\title{
Distribución potencial de dos subespecies de Montanoa tomentosa (Asteracerae) en México
}

\author{
Néstor Hernández-Silva ${ }^{1}$, Jesús Hernández-Ruiz ${ }^{2}$, M. Socorro Gonzalez-Elizondo ${ }^{3}$, \\ Rodolfo Figueroa-Brito $^{1 *} \&$ Nemesio Villa-Ruano ${ }^{4}$
}

1. Centro de Desarrollo de Productos Bóticos, Instituto Politécnico Nacional, Carretera Yautepec-Jojutla, Km. 6, calle CEPROBI No. 8, Col. San Isidro, Yautepec, Morelos, México; nhsceprobi@outlook.com, xiuhcoatl@live.cl

2. División de Ciencias de la Vida, Universidad de Guanajuato, Carretera Irapuato-Silao, km 9, Ex Hacienda El Copal, Irapuato, Guanajuato, México; hernandez.jesus@ugto.mx

3. Centro Interdisciplinario de Investigación para el Desarrollo Integral Regional Durango, Instituto Politécnico Nacional, Sigma 119 Frac. 20 de Noviembre II, Durango Dgo., México; herbario_ciidir@yahoo.com.mx

4. Centro Universitario de Vinculación y Transferencia de Tecnología, Benemérita Universidad Autónoma de Puebla, Prol. 24 Sur, Cdad. Universitaria Puebla, México; necho82@yahoo.com.mx

* Correspondencia

Recibido 25-IX-2019. Corregido 12-VI-2020. Aceptado 06-V-2020.

\begin{abstract}
Potential distribution of two subspecies of Montanoa tomentosa (Asteracerae) in Mexico. Introduction: Montanoa tomentosa is a phytogenetic resource with medicinal properties used in Mexico. Despite its botanic relevance, the specific information of infraespecific taxa distributions and the environmental factors that influence their development is still limited. Objective: To report the potential geographic distribution and the environmental variables associated to ecological niches of two subspecies of $M$. tomentosa (M. tomentosa subsp. tomentosa and M. tomentosa subsp. xanthiifolia) in the Mexican territory. Methods: A database of herbarium records was created, an in situ systematic search of both subspecies was carried out from October 2015 to March 2016, during the flowering-fruiting season. After that, exploratory tours (Guanajuato, Querétaro, Hidalgo, Mexico City, State of Mexico, Puebla, Oaxaca and Chiapas) were realized. A total of 181 individuals were sampled and georeferenced, generating new records for the database upgrading. Three habitat suitability models (M. tomentosa complex, M. tomentosa subsp. tomentosa and M. tomentosa subsp. xanthiifolia) were generated using the MaxEnt software. These software estimates the probability of species distribution using geo-referenced data and environmental characteristics as predictors, through the maximum entropy logarithm and the Bayesian method. Results: From 22 environmental variables used as predictors, five (seasonality of temperature, isothermality, maximum temperature of the warmest month, precipitation of the warmest trimester and precipitation of the wettest trimester) contributed $70.2 \%$ of the total percentage contribution. The model showed a potential distribution of $77688 \mathrm{~km}^{2}$, approximately $4 \%$ of the Mexican Republic territory. Both subspecies grew between 500 and $2500 \mathrm{~m}$. They were associated to xerophilous and heliophiles vegetation, sometimes they were located in the periphery or embed in openings of temperate and sub-humid forests too. They were not present dry regions from the North of the country. Conclusions: Temperature seasonality, isothermality and maximum temperature of the warmest month determine the distribution of the species. Precipitation of the warmest quarter and precipitation of wettest quarter defines the habitat between M. tomentosa subsp. tomentosa and $M$. tomentosa subsp. xanthiifolia. These findings clarify the ecological preferences and delimitation the infraspecific taxa of $M$. tomentosa.
\end{abstract}

Key words: ecological niche; habitat suitability; heliophile; infraspecific taxa; MaxEnt; zoapatle.

Hernández-Silva, N., Hernández-Ruiz, J., Gonzalez-Elizondo, M.S., Figueroa-Brito, R., \& VillaRuano, N. (2020). Distribución potencial de dos subespecies de Montanoa tomentosa (Asteracerae) en México. Revista de Biología Tropical, 68(4), 1095-1106. 
El género Montanoa Cerv. (Asteraceae) representa una fuente importante de extracción, síntesis y biosíntesis de metabolitos secundarios de interés farmacológico, con más de 70 productos naturales registrados (Villa-Ruano \& Lozoya-Gloria, 2014). Montanoa tomentosa Cerv., comúnmente conocida como "zoapatle" por las raíces cihutal (mujer) y pathli (medicina), es una planta neotropical utilizada en la medicina tradicional mexicana como auxiliar en partos, regulador del ciclo menstrual, analgésico y anticonceptivo (Hahnn, Ericson, Lay \& Probst, 1981; Lozoya-Legorreta, VelázquezDíaz, \& Flores-Alvarado, 1988; Carro-Juárez, Franco \& Rodríguez-Peña, 2014). Es considerada recurso fitogenético no alimenticio por la Food and Agriculture Organization (FAO) (Molina-Moreno \& Córdoba-Téllez, 2006). $\mathrm{Su}$ uso medicinal se atribuye a la actividad uterotónica y uterocontractora de los compuestos mayoritarios ácidos kaurenoico, monogenoico y grandiflorénico (Robles-Zepeda et al., 2006; Villa-Ruano, Betancourt-Jiménez \& Lozoya-Gloria, 2009).

Estudios sistemáticos fundamentados en caracteres morfológicos de estructuras vegetativas (hojas y brácteas) y reproductivas (capítulos, flores y aquenios) agruparon a la especie en cuatro taxa infraespecíficos $[M$. tomentosa subsp. microcephala (Sch. Bip. ex K. Koch) V.A. Funk; M. tomentosa subsp. rosei (Rose ex B.L. Rob. \& Greenm.) V.A. Funk; $M$. tomentosa subsp. tomentosa y M. tomentosa subsp. xanthiifolia (Sch. Bip. ex K. Koch) V.A. Funk] (Funk, 1982). Trabajos filogenéticos posteriores no mostraron diferencias entre las subespecies tomentosa, microcephala y xanthiifolia y no contemplaron la subespecie rosei (Plovanich \& Paner, 2004), la cual actualmente se reclasificó a nivel de especie (Martin et al., 1998; van Devender, Sanders, Wilson \& Meyer, 2000; Panero \& Villaseñor, 2008).

En general, las variantes infraespecíficas de los organismos son resultado de procesos evolutivos (Manel, Schwartz, Luikart, \& Taberlet, 2003), influenciados entre otros factores por la estructura geográfica y ambiental del paisaje en que se distribuyen (Paiaro, Oliva,
Cocucci, \& Sérsic, 2012). Esta interacción genera cambios en la formación y concentración de los productos naturales (Külheim et al., 2011), como ha sido registrado en otras plantas (Zhao et al., 2003; Chen et al., 2013; Chen et al., 2015).

La distribución de las especies puede calcularse mediante modelamientos predictivos con Sistemas de Información Geográfica (SIG) (Guisan \& Thuiller, 2005). Estos modelos permiten generar estimaciones de nicho ecológico e indirectamente de distribución, basados en localidades de recolecta y observación georreferenciadas y variables ambientales como predictores (Phillips, Dudík \& Schapire, 2019). Por lo anterior, este trabajo tuvo como objetivo estimar la distribución geográfica de $M$. tomentosa subsp. tomentosa y M. tomentosa subsp. xanthiifolia, así como identificar las variables ambientales asociadas a la formación de sus nichos ecológicos, dentro del territorio mexicano.

\section{MATERIALES Y MÉTODOS}

Base de datos: Se elaboró una matriz de datos de registros de $M$. tomentosa subsp. tomentosa y $M$. tomentosa subsp. xanthiifolia a partir de la base del Herbario Nacional del Instituto de Biología de la Universidad Nacional Autónoma de México (IBUNAM: MEXU) (Apéndice Digital 1), complementada con registros utilizados en la revisión más extensa del género Montanoa (Funk, 1982) y otros obtenidos durante el desarrollo de este trabajo. Para estos últimos, las coordenadas se tomaron con un receptor de posicionamiento satelital GPS Garmin ETrex (Datum ITRF92). La georreferenciación, para casos de registros previos sin ubicación exacta, fue generada mediante Google Earth. Se realizaron recorridos de campo en diferentes localidades de los estados de Guanajuato, Querétaro, Hidalgo, Ciudad de México, Estado de México, Puebla, Oaxaca y Chiapas durante la etapa de floración y fructificación (octubre 2015 a marzo 2016). Las exploraciones fueron planeadas para cubrir la mayor parte de las regiones donde 
se han documentado registros de esta especie. Se lograron obtener 181 nuevos registros (Apéndice Digital 2). Esta información complementó la base de datos históricos inicial, con lo que se obtuvo un total de 314 registros.

Modelado de áreas potenciales de distribución: Se generó un modelo de idoneidad del hábitat con el software MaxEnt versión 3.4.1, el cual estima la probabilidad de distribución de las especies basada en el conjunto de localidades de recolecta y observación georreferenciadas y sus variables ambientales como predictores, mediante el método de máxima entropía y el método bayesiano (Phillips et al., 2019). Se utilizaron 22 variables (Tabla 1), 19 bioclimáticas con resolución espacial de 0.5 min de arco, obtenidas de la base de datos WorldClim (www.worldclim.org). Los datos digitales de elevación (DEM, $90 \mathrm{~m}$ de resolución) se obtuvieron de CGIAR-CSI (http:// srtm.csi.cgiar.org), mientras que las capas en formato vectorial de uso de suelo y vegetación se tomaron de la Comisión Nacional para el Conocimiento y Uso de la Biodiversidad (INEGI, 2013). El régimen de humedad del suelo también se adquirió de la misma comisión (Maples-Vermeersch, 1992).

\section{RESULTADOS}

Análisis del nicho ecológico de Montanoa tomentosa en México: Se realizaron tres modelos de distribución con el programa MaxEnt 3.4.1. El primer modelo abarcó las dos subespecies estudiadas (Fig. 1A), el segundo se elaboró solo para $M$. tomentosa subsp. tomentosa (Fig. 1B) y el tercero para

TABLA 1

Variables ambientales y bioclimáticas utilizadas para determinar la distribución geográfica de Montanoa tomentosa subsp. tomentosa y Montanoa tomentosa subsp. xanthiifolia en México

TABLE 1

Environmental and bioclimatic variables used to determine the geographic distribution of Montanoa tomentosa subsp. tomentosa and Montanoa tomentosa subsp. xanthiifolia in Mexico

\begin{tabular}{lc}
\multicolumn{1}{c}{ Descripción de la variable } & Unidades * \\
Temperatura media anual & ${ }^{\circ} \mathrm{C}$ \\
Ámbito de temperatura media diurna & ${ }^{\circ} \mathrm{C}$ Adimensional \\
Isotermalidad & $\mathrm{CV}$ \\
Estacionalidad de la temperatura & ${ }^{\circ} \mathrm{C}$ \\
Temperatura máxima del mes más cálido & ${ }^{\circ} \mathrm{C}$ \\
Temperatura mínima del mes más frío & ${ }^{\circ} \mathrm{C}$ \\
Ámbito anual de temperatura & ${ }^{\circ} \mathrm{C}$ \\
Temperatura media del trimestre más lluvioso & ${ }^{\circ} \mathrm{C}$ \\
Temperatura media del trimestre más seco & ${ }^{\circ} \mathrm{C}$ \\
Temperatura media del trimestre más cálido & ${ }^{\circ} \mathrm{C}$ \\
Temperatura media del trimestre más frío & $\mathrm{mm}$ \\
Precipitación anual & $\mathrm{mm}$ \\
Precipitación del mes más húmedo & $\mathrm{mm}$ \\
Precipitación del mes más seco & $\mathrm{CV}$ \\
Estacionalidad de la precipitación & $\mathrm{mm}$ \\
Precipitación del trimestre más lluvioso & $\mathrm{mm}$ \\
Precipitación del trimestre más seco & $\mathrm{mm}$ \\
Precipitación del trimestre más cálido & $\mathrm{mm}$ \\
Precipitación del trimestre más frío & $\mathrm{m}$ \\
Altitud & ${ }^{\circ}$ \\
Régimen de humedad & $23 \mathrm{tipos}$ \\
Vegetación & \\
\hline
\end{tabular}

$* \circ \mathrm{C}=$ grados centígrados, $\mathrm{CV}=$ coeficiente de variación, $\mathrm{m}=$ metros, $\mathrm{mm}=$ milímetros. 


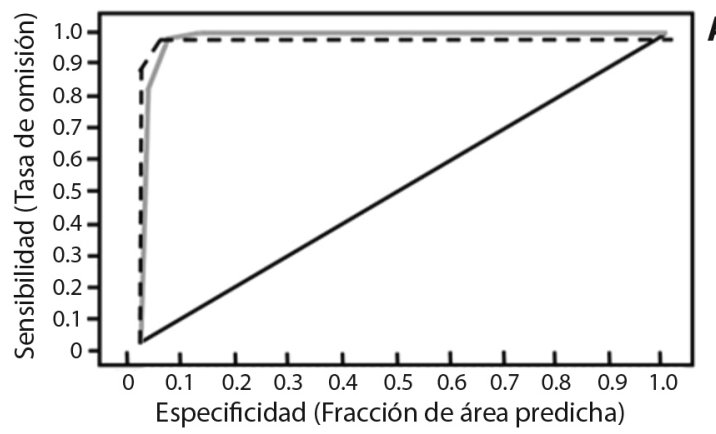

Datos de prueba (AUC $=0.988$ )

-1. Comprobación de datos $(A U C=0.992)$

Predicción al azar (AUC $=0.5$ )

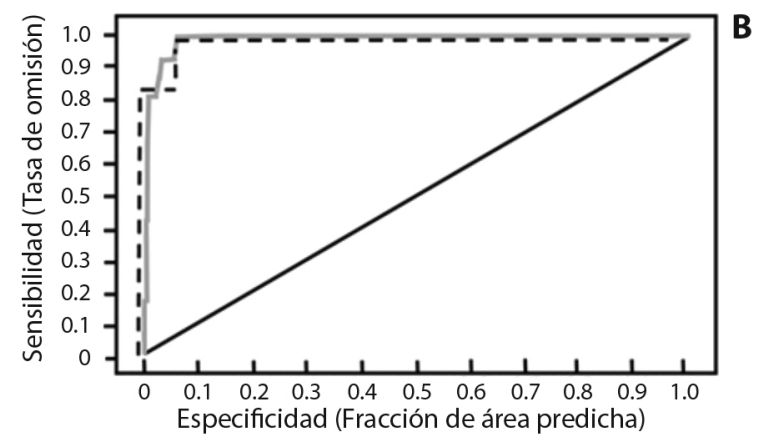

- Datos de prueba ( $A \cup C=0.990)$

-m Comprobación de datos (AUC = 0.996)

Predicción al azar (AUC = 0.5)

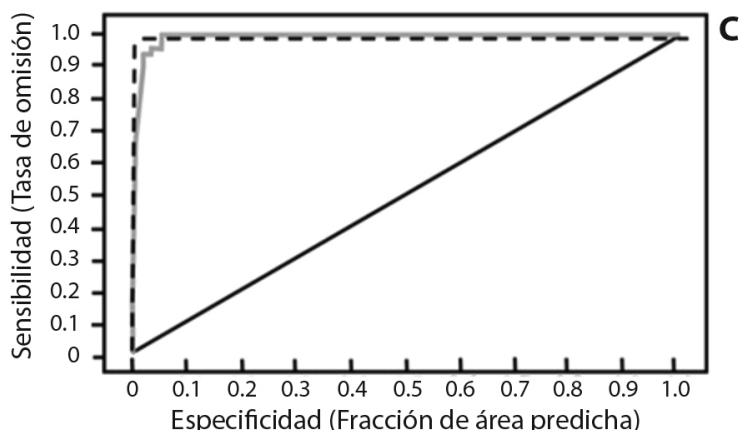

specificidad (Fracción de área predicha)

Datos de prueba $($ AUC $=0.991)$

-1- Comprobación de datos (AUC = 0.998)

Predicción al azar (AUC $=0.5)$

Fig. 1. Sensibilidad y especificidad para los modelos de Montanoa tomentosa. La línea gris muestra el ajuste del modelo a los datos de información de la especie. La línea discontinua indica el ajuste del modelo a los datos de prueba. La sensibilidad frente a especificidad se muestra para A. dos subespecies estudiadas, B. M. tomentosa subsp. tomentosa y C. M. tomentosa subsp. xanthiifolia.

Fig. 1. Sensitivity and specificity for Montanoa tomentosa models. The gray line shows the fit of the model to the information data of the species. The dashed line indicates the fit of the model to the test data. Sensitivity to specificity is shown for A. two subspecies studied, B. M. tomentosa subsp. tomentosa and C. M. tomentosa subsp. xanthiifolia. 

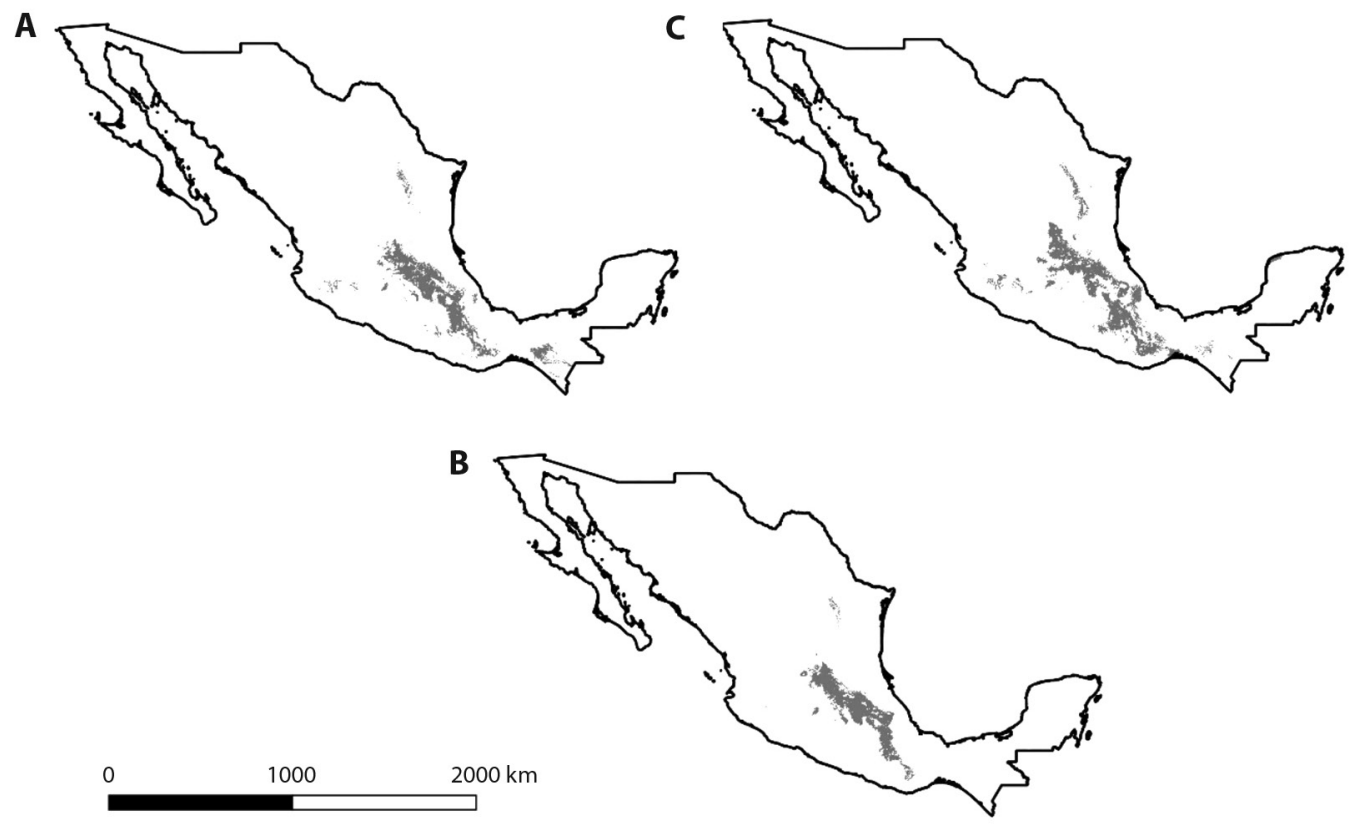

Fig. 2. Distribución potencial de Montanoa tomentosa: A. dos subespecies, B. subsp. tomentosa y C. subsp. xanthiifolia, determinada a través del modelo de máxima entropía. En negro se observan las zonas de mayor potencial de distribución. Fig. 2. Potential distribution of Montanoa tomentosa: A. two subspecies, B. subsp. tomentosa and C. subsp. xanthiifolia, determined through the maximum entropy model. In black, the areas of greatest distribution potential are observed.

M. tomentosa subsp. xanthiifolia (Fig. 1C). Los valores del área bajo la curva del umbral acumulativo (AUC) para los datos de prueba de cada modelo fueron $0.988,0.990$ y 0.991 , respectivamente. Asimismo, los valores de AUC para la comprobación de datos en cada modelo fueron $0.992,0.996$ y 0.998 . Se obtuvo el valor máximo para la predicción al azar en todos los casos (0.5).

El área de máxima entropía calculada para las dos subespecies fue de aproximadamente $77688 \mathrm{~km}^{2}$, que representa $4 \%$ del territorio de la República Mexicana (Fig. 2A, 2B y 2C).

Variables climáticas: De las 19 variables bioclimáticas utilizadas como predictores para el complejo de $M$. tomentosa, cinco contribuyeron con $70.2 \%$ de la estimación total (Tabla 2).

En regiones del país con variación estacional de la temperatura de 14 a $26 \%$ y precipitación media en el trimestre más cálido de 200 $\mathrm{mm}$ y de $300 \mathrm{~mm}$ en el trimestre más lluvioso, existe mayor probabilidad de presencia de la especie (por arriba de 0.6) (Fig. 3A, 3B y 3C). Además, en zonas donde la temperatura es superior a $30{ }^{\circ} \mathrm{C}$ durante el mes más cálido, la probabilidad disminuye hasta 0.3 (Fig. 3D). Los

\section{TABLA 2}

Contribuciones porcentuales de las principales cinco variables bioclimáticas en el modelo MaxEnt para Montanoa tomentosa subsp. tomentosa y Montanoa tomentosa subsp. xanthiifolia en México

TABLE 2

Percentage contributions of the main five bioclimatic variables in the MaxEnt model for Montanoa tomentosa subsp. tomentosa and Montanoa tomentosa subsp. xanthiifolia in Mexico

\begin{tabular}{lc}
\multicolumn{1}{c}{ Variable } & $\begin{array}{c}\text { Contribución } \\
\%\end{array}$ \\
\hline Estacionalidad de la temperatura & 21.8 \\
Isotermalidad & 17.7 \\
Temperatura máxima del mes más cálido & 13.2 \\
Precipitación del trimestre más cálido & 9.6 \\
Precipitación del trimestre más lluvioso & 7.9 \\
Total & 70.2 \\
\hline
\end{tabular}



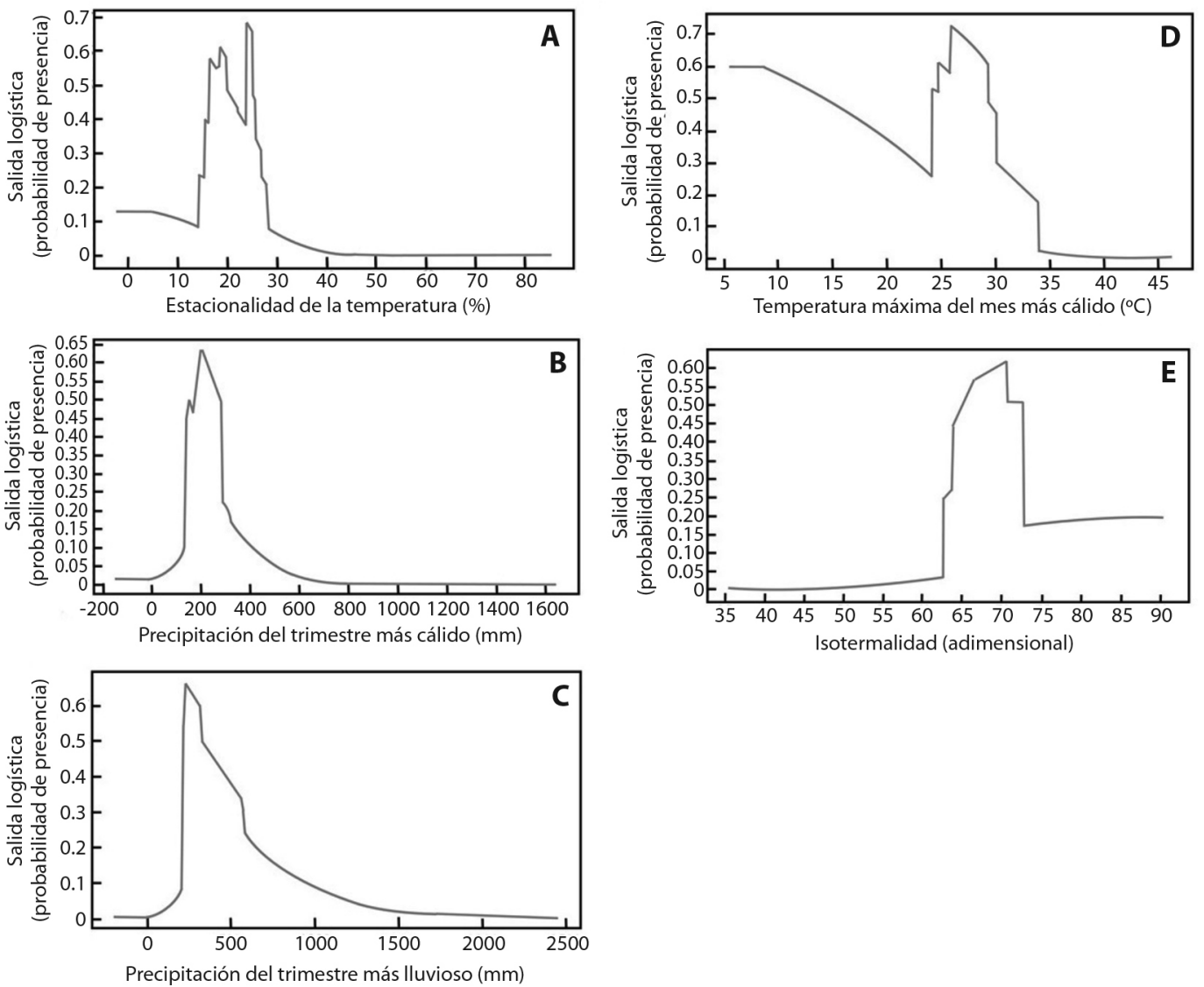

Fig. 3. Variables bioclimáticas de mayor contribución en el modelamiento de máxima entropía para el conjunto $M$. tomentosa subsp. tomentosa y M. tomentosa subsp. xanthiifolia. A. Estacionalidad de la temperatura, B. Precipitación del trimestre más cálido, C. Precipitación del trimestre más lluvioso, D. Temperatura máxima del mes más cálido, E. Isotermalidad.

Fig. 3. Bioclimatic variables of greater contribution in the modeling of maximum entropy for the set M. tomentosa subsp. tomentosa and M. tomentosa subsp. xanthiifolia A. Seasonality of temperature, B. Precipitation of the warmest quarter, C. Precipitation of the wettest quarter, D. Maximum temperature of the warmest month, E. Isothermality.

valores de isotermalidad (variación de la temperatura) óptimos para el desarrollo de ambas subespecies son de 63 a 72 unidades (Fig. 3E).

Análisis por subespecie: Las variables de mayor impacto en el modelo de distribución de M. tomentosa subsp. tomentosa fueron precipitación en el trimestre más frío, isotermalidad y temperatura estacional. En zonas sin precipitación en el trimestre más frío, la probabilidad de presencia de la subespecie es superior a 0.9 y disminuye hasta 0.0 en regiones con precipitaciones superiores a $50 \mathrm{~mm}$ (Fig. 4A).
Zonas con valores isotérmicos de 85 unidades tienen una probabilidad de presencia superior a 0.9 , la cual disminuye hasta 0.2 en zonas con 60 unidades (Fig. 4B). En regiones donde la temperatura estacional es menor o igual a $10 \%$ se estima una probabilidad de presencia de 1.0 de la subespecie tomentosa, la cual disminuye hasta 0.1 en regiones donde la estacionalidad de la temperatura es superior a $30 \%$ (Fig. 4C).

Las variables de mayor impacto en el modelo de distribución de M. tomentosa subsp. xanthiifolia fueron la estacionalidad de la temperatura, la isotermalidad y el ámbito anual 

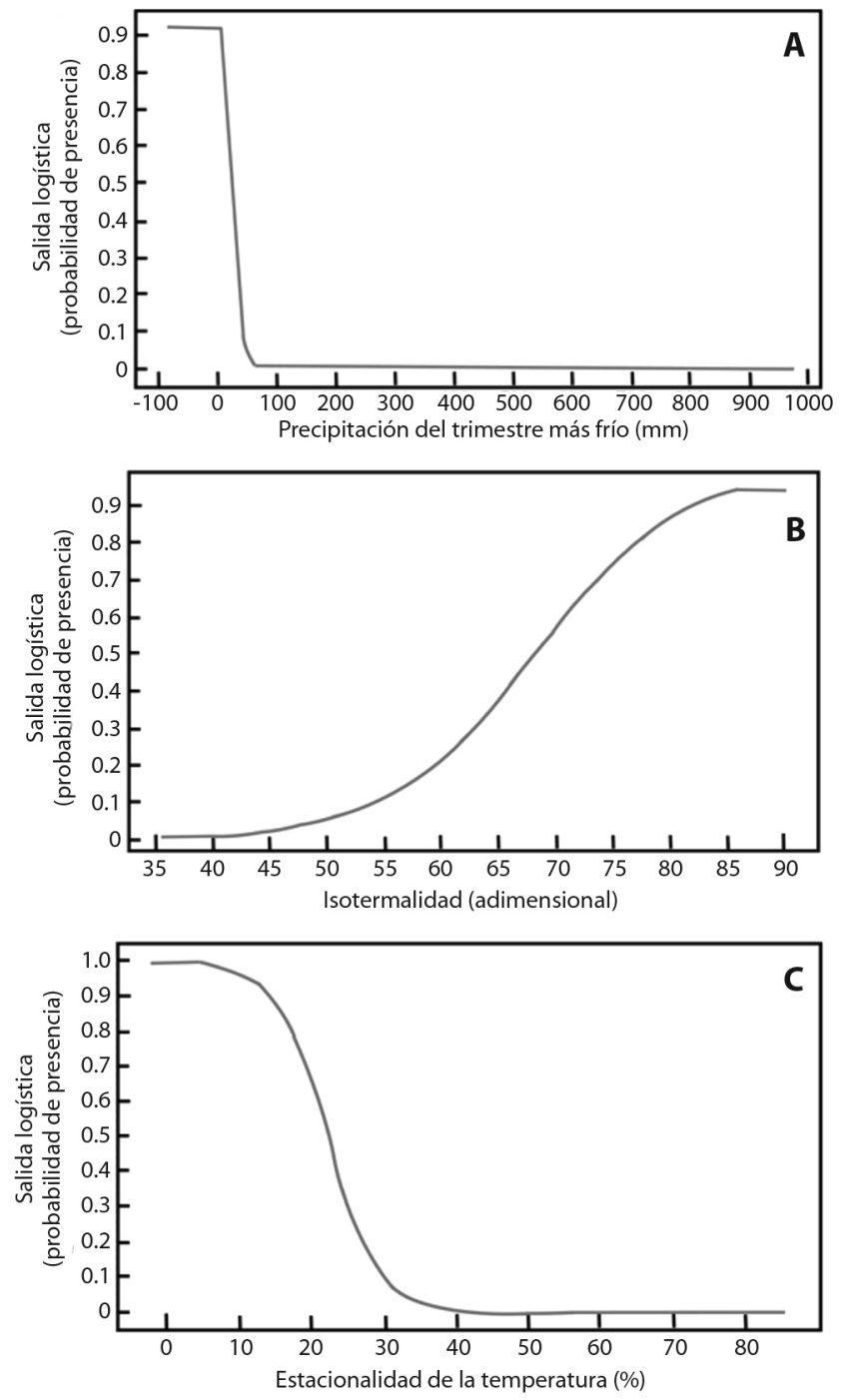

Fig. 4. Variables bioclimáticas de mayor contribución en el modelamiento de máxima entropía para M. tomentosa subsp. tomentosa. A. Precipitación del trimestre más frío, B. Isotermalidad, C. Estacionalidad de la temperatura.

Fig. 4. Bioclimatic variables of greater contribution in the modeling of maximum entropy for M. tomentosa subsp. tomentosa A. Precipitation of the coldest quarter, B. Isothermality, C. Seasonality of temperature.

de temperatura. En zonas con $20 \%$ de variación en la temperatura estacional, la probabilidad de presencia de la subespecie es superior a 0.75 y disminuye a 0.2 en zonas con variación inferior o igual a $10 \%$ y superior o igual a $30 \%$ (Fig. 5A). En regiones con isotermalidad de 65 unidades existe una probabilidad de distribución de 0.6 , la cual se reduce hasta 0.05 en zonas con valores por debajo de 55 o superiores a 85 unidades (Fig. 5B). En regiones con temperaturas que oscilan $23^{\circ} \mathrm{C}$, la probabilidad de presencia de la especie es de 0.6 y disminuye hasta 0.1 en regiones donde el ámbito supera $28^{\circ} \mathrm{C}$ (Fig. 5C).

Otras variables: El complejo de $M$. tomentosa estudiado se ubica a una altitud que va de 500 a $2500 \mathrm{~m}$, pero con mayor frecuencia 

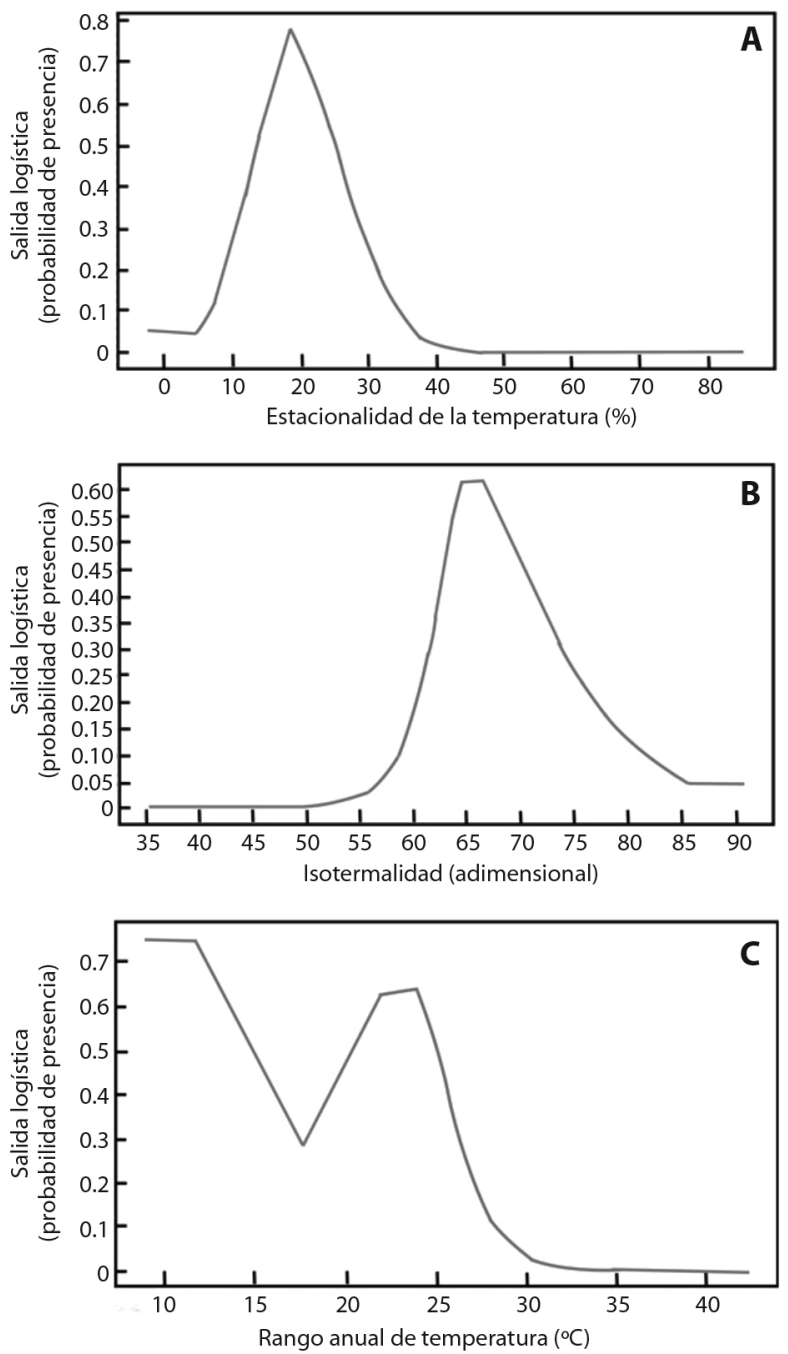

Fig. 5. Variables bioclimáticas de mayor contribución en el modelamiento de máxima entropía para M. tomentosa subsp. xanthiifolia. A. Estacionalidad de la temperatura, B. Isotermalidad, C. Ámbito anual de temperatura.

Fig. 5. Bioclimatic variables of greater contribution in the modeling of maximum entropy for M. tomentosa subsp. xanthiifolia A. Temperature seasonality, B. Isothermality, C. Annual temperature range.

en un recorrido de 1500 a $2500 \mathrm{~m}$. Los tipos de vegetación a los que mejor se asocia son la vegetación xerófila (matorrales desérticos y crasicaules), zonas con clima subhúmedo, ocupando la periferia y los claros de bosque tropical perennifolio y subcaducifolio, bosques de coníferas, bosques de latifoliadas y bosque mesófilo de montaña.

El régimen de humedad del suelo (RHS) predominante en los hábitats de esta especie es xérico y ústico (90 a 180 y 180 a 270 días de suelo húmedo, respectivamente), con 
precipitaciones de 400 a $1200 \mathrm{~mm}$ y en menor probabilidad con RHS arídico y údico (menos de 90 y 270 a 330 días de suelo húmedo, respectivamente), con precipitaciones de 125 a $400 \mathrm{~mm}$ y de 1200 a $2000 \mathrm{~mm}$.

\section{DISCUSIÓN}

La base de datos de M. tomentosa, utilizada en los modelos de distribución, se generó a partir de reportes de presencia históricos y de visitas recientes a los sitios registrados de recolecta y observación. Las nuevas poblaciones georreferenciadas fueron seleccionadas de acuerdo con las claves sistemáticas utilizadas por Funk (1982). Esta información representa el registro más actualizado para ambas subespecies. El ámbito geográfico que abarcan los datos presentados cubre las condiciones ecológicas del área geográfica en que las subespecies pueden desarrollarse. Por esta razón, los resultados coinciden en gran medida con la distribución documentada previamente por Funk (1982).

El área bajo la curva de los tres modelamientos registró valores de AUC cercanos a la unidad, tanto para los datos de prueba como para la comprobación de datos. Los valores de AUC varían de 0 a 1 , de tal modo que los superiores a 0.9 se clasifican como excelentes y los menores o iguales a 0.6 como inválidos (Hoffman, Narumalani, Mishra, Merani, \& Wilson, 2008). Por esta razón, se considera que la capacidad predictiva de los modelamientos generados es apropiada. En este sentido, Elham, Mostafa, Mehdi y Mohammad (2015) mencionan que los valores de AUC tienden a ser menores para las especies con una amplia distribución geográfica.

Generalmente, los patrones de distribución son resultado de interacciones bióticas y abióticas, además de factores históricos ambientales y orográficos (Maciel-Mata, Manríquez-Morán, Octavio-Aguilar \& SánchezRojas, 2015), los cuales no son considerados por los algoritmos de modelado, pero pueden proporcionar información valiosa sobre orígenes históricos, relaciones con otras especies y capacidad potencial de colonización (Peterson, Ball, \& Cohoon, 2002). Por ejemplo, el hábitat de $M$. tomentosa suele ubicarse en espacios abiertos, perturbados por el hombre, como asentamientos humanos, caminos o veredas y zonas de producción agrícola con remoción regular del suelo. Estas características son propias de especies con hábito de crecimiento ruderal y arvense (Martínez-De la Cruz et al., 2015), predominantemente heliófilas.

Lo anterior se observa en la enorme cantidad de especímenes de $M$. tomentosa recolectados en hábitats perturbados, de tal modo que el modelado de nichos ecológicos debe interpretarse como la representación geográfica de las condiciones ambientales requeridas por la especie, como han sugerido Peterson (2003) y Martínez-Meyer (2005) para otros organismos. Asimismo, los resultados mostraron zonas potenciales para el desarrollo de las dos subespecies, aunque no existe información biológica que respalde dicha distribución. Esto podría deberse a la ausencia de recolectas en estos sitios, como ha ocurrido con otras especies (Luna-Vega, Alcántara-Ayala, ContrerasMedina, \& Ríos-Muñoz, 2012). Además, los modelos tanto de nicho ecológico idóneo como de amplitud de distribución de especies son de tipo probabilístico (Broennimann et al., 2006) y las especies no están presentes en todas las áreas potenciales de distribución, como lo destacan Soberón y Peterson (2005).

En este trabajo se amplía la información presentada por Funk (1982) sobre el tipo de vegetación y el gradiente altitudinal de la zona en que se desarrolla $M$. tomentosa y se establecen las características ambientales que favorecen la idoneidad de su hábitat. Funk (1982) menciona poblaciones atípicas de M. tomentosa subsp. xanthiifolia localizadas a $230 \mathrm{~m}$ y registros de $M$. tomentosa subsp. tomentosa a elevaciones mayores de $2800 \mathrm{~m}$. Estos datos difieren de los resultados obtenidos en el presente trabajo. El registro a muy baja elevación parece corresponder a un taxón diferente, por lo que los especímenes probablemente requieren de una revisión adicional. 
En México, la mayor riqueza de especies de la familia Asteraceae se localiza en los bosques mesófilo, templado y tropical (CruzCárdenas, Villaseñor, López-Mata, \& Ortiz, 2013). En el caso particular de M. tomentosa, se observó que la presencia de ambas subespecies se limita a zonas de clima seco con matorral xerófilo desértico y crasicaule en el centro y sur del país, así como a comunidades abiertas dentro de zonas con predominancia templada, tropical y del bosque mesófilo de montaña (claros desprovistos de vegetación y periferia de esos bosques).

Los resultados obtenidos en el presente trabajo aclaran las principales características ambientales que delimitan el hábitat de $M$. tomentosa. La estacionalidad de la temperatura, isotermalidad y temperatura máxima del mes más cálido determinan la distribución de la especie. La precipitación del trimestre más cálido y la precipitación del trimestre más lluvioso definen el hábitat entre ambas subespecies al observarse un efecto negativo en la distribución de $M$. tomentosa subsp. tomentosa respecto a regiones con mayor precipitación.

Declaración de ética: los autores declaran que todos están de acuerdo con esta publicación y que han hecho aportes que justifican su autoría; que no hay conflicto de interés de ningún tipo; y que han cumplido con todos los requisitos y procedimientos éticos y legales pertinentes. Todas las fuentes de financiamiento se detallan plena y claramente en la sección de agradecimientos. El respectivo documento legal firmado se encuentra en los archivos de la revista.

\section{AGRADECIMIENTOS}

A Vicki A. Funk y José L. Panero por el envío de literatura e información. Al CONACYT, México por la beca otorgada a Néstor Hernández Silva para estudios de posgrado. El Instituto Politécnico Nacional proporcionó apoyo logístico para el desarrollo del trabajo.

\section{RESUMEN}

Introducción: Montanoa tomentosa es un recurso fitogenético con propiedades medicinales utilizada en México. A pesar de su relevancia para la botánica, la información específica sobre la distribución de sus taxa infraespecíficos y los factores ambientales que influyen en su desarrollo aún es limitada. Objetivo: Informar sobre la distribución geográfica potencial y las variables ambientales asociadas a los nichos ecológicos de dos subespecies de $M$. tomentosa (M. tomentosa subsp. tomentosa y M. tomentosa subsp. xanthiifolia), dentro del territorio mexicano. Métodos: Se elaboró una base de datos con registros de herbario. Posteriormente, se realizó una búsqueda sistemática in situ de plantas de ambas subespecies entre octubre 2015 y marzo 2016 durante la época de floración-fructificación. Se realizaron recorridos exploratorios en los estados de Guanajuato, Querétaro, Hidalgo, Ciudad de México, Estado de México, Puebla, Oaxaca y Chiapas. Un total de 181 plantas fueron ubicadas y georreferenciadas. La base de datos se complementó con los nuevos registros (un total de 314). Se generaron tres modelos de idoneidad del hábitat (complejo de M. tomentosa, M. tomentosa subsp. tomentosa y M. tomentosa subsp. xanthiifolia) con el software MaxEnt, el cual estima la probabilidad de distribución de especies a partir de datos georreferenciados y características ambientales como predictores, mediante el logaritmo de máxima entropía y el método bayesiano. Resultados: De 22 variables ambientales utilizadas como predictores, cinco (estacionalidad de la temperatura, isotermalidad, temperatura máxima del mes más cálido, precipitación del trimestre más cálido y precipitación del trimestre más lluvioso) contribuyeron con $70.2 \%$ de la estimación total. El modelo arrojó una distribución potencial de 77688 km² aproximadamente, $4 \%$ del territorio de la república mexicana. Ambas subespecies crecen a una altitud entre 500 y $2500 \mathrm{~m}$. Las heliófilas se asocian a vegetación xerófila y a veces se localizan en la periferia de bosques o enclavadas en claros de bosques templados y subhúmedos, pero no habitan en las regiones áridas y semiáridas del norte del país. Conclusiones: La estacionalidad de la temperatura, isotermalidad y temperatura máxima del mes más cálido determinan la distribución de la especie. La precipitación en el trimestre más cálido y la precipitación en el trimestre más lluvioso definen el hábitat entre $M$. tomentosa subsp. tomentosa y M. tomentosa subsp. xanthiifolia. Estos hallazgos aclaran las preferencias ecológicas y la delimitación de los taxa infraespecíficos de $M$. tomentosa.

Palabras clave: nicho ecológico; idoneidad del hábitat; heliófila; taxa infraespecíficos; MaxEnt; zoapatle.

\section{REFERENCIAS}

Broennimann, O., Thuiller, W., Hughes, G., Midgley, G.F., Alkemade, J.M.R., \& Guisan, A. (2006). Do geographic distribution, niche property and life form 
explain plants' vulnerability to global change? Global Change Biology, 12(6), 1079-1093.

Carro-Juárez, M., Franco, M.A., \& Rodríguez-Peña, M.L. (2014). Increase of the Ejaculatory Potency by the Systemic Administration of Aqueous Crude Extracts of Cihuapatli (Montanoa Genus) Plants in Spinal Male Rats. Journal of Evidence-Based Complementary and Alternative Medicine, 19(1), 43-50.

Chen, J., Li, Z., Maiwulanjiang, M., Zhang, W.L., Zhan, J.Y., Lam, C.T., \& Tsim, K.W. (2013). Chemical and biological assessment of Ziziphus jujuba fruits from China: different geographical sources and developmental stages. Journal of Agricultural and Food Chemistry, 61, 7315-7324.

Chen, J., Xu, Y., Wei, G., Liao, S., Zhang, Y., Huang, W., \& Wang, Y. (2015). Chemotypic and genetic diversity in Epimedium sagittatum from different geographical regions of china. Phytochemistry, 116, 180-187.

Cruz-Cárdenas, G., Villaseñor, J.L., López-Mata, L., \& Ortiz, E. (2013). Distribución espacial de la riqueza de especies de plantas vasculares en México. Revista Mexicana de Biodiversidad, 84, 1189-1199.

Elham, G., Ardestani, M.T., Mehdi, B., \& Mohammad, R.V. (2015). Potential habitat modeling for reintroduction of three native plant species in central Iran. Journal of Arid Land, 7(3), 381-390.

Funk, V.A. (1982). The Systematics of Montanoa (Asteraceae, Heliantheae). Memoirs of The New York Botanical Garden, 36, 1-135.

Guisan, A., \& Thuiller, W. (2005). Predicting species distribution: offering more than simple habitat models. Ecology Letters, 8, 993-1009.

Hahnn, D.W., Ericson, E.W., Lai, M.T., \& Probst, A. (1981). Antifertility activity of Montanoa tomentosa (Zoapatle). Contraception, 23, 133-140.

Hoffman, J.D., Narumalani, S., Mishra, D.R., Merani, P., \& Wilson, R.G. (2008). Predicting potential occurrence and spread of invasive plant species along the North Platte River, Nebraska. Invasive Plant Science and Management, 1, 359-367.

Instituto Nacional de Estadística y Geografía (INEGI) (2013). Conjunto de Datos Vectoriales de Uso de Suelo y Vegetación. Escala 1:250 000. Serie V (Capa Unión), escala: 1:250 000. Edición: 1. Aguascalientes, México: Instituto Nacional de Estadística y Geografía.

Külheim, C., Yeoh, S.H., Wallis, I.R., Laffan, S., Moran, G.F., \& Foley, W.J. (2011). The molecular basis of quantitative variation in foliar secondary metabolites in Eucalyptus globulus. New Phytologist, $191,1041-1053$
Lozoya-Legorreta, X., Velázquez-Diaz, G., \& Flores-Alvarado, A. (1988). La medicina tradicional en México: Experiencia del programa IMSS-COPLAMAR 19821987. Ciudad de México, México: Instituto Mexicano del Seguro Social (IMSS).

Luna-Vega, I., Alcántara-Ayala, O., Contreras-Medina, R. \& Ríos-Muñoz, C.A. (2012). Ecological niche modeling on the effect of climatic change and conservation of Ternstroemia lineata DC. (Ternstroemiaceae) in Mesoamerica. Botany, 90, 637-650.

Manel, S., Schwartz, M.K., Luikart, G., \& Taberlet, P. (2003). Landscape genetics: combining landscape ecology and population genetics. Trends in Ecology and Evolution, 18(4), 189-197.

Maples-Vermeersch, M. (1992). Regímenes de humedad del suelo en Hidrogeografía IV.6.2 Atlas Nacional de México. Vol. II. Escala 1:4000000. Ciudad de México, México: Instituto de Geografía, Universidad Nacional Autónoma de México.

Martin, P.S., Yetman, D.A., Fishbein, M.E., Jenkins, P.D., van Devender, T.R., \& Wilson, R.K. (1998). Gentry's Rio Mayo Plants: The Tropical Deciduous Forest and Environs of Northwest Mexico. Tucson Arizona: The University of Arizona Press.

Martínez-De la Cruz, I., Vibrans, H., Lozada-Pérez, L., Romero-Manzanares, A., Aguilera-Gómez, L.I., \& Rivas-Manzano, I.V. (2015). Plantas ruderales del área urbana de Malinalco, Estado de México, México. Botanical Sciences, 93(4), 907-919.

Martínez-Meyer, E. (2005). Climate change and biodiversity: some considerations in forecasting shifts in species' potential distributions. Biodiversity Informatics, 2, 42-55.

Maciel-Mata, C.A., Manríquez-Morán, N., Octavio-Aguilar, P., \& Sánchez-Rojas, G. (2015). El área de distribución de las especies: revisión del concepto. Acta Universitaria, 25(2), 3-19.

Molina-Moreno, J.C., \& Córdova-Téllez, C.T. (2006) Recursos Fitogenéticos de México para la Alimentación y la Agricultura: Informe Nacional 2006. Texcoco, Estado de México, México: Secretaría de Agricultura, Ganadería, Desarrollo Rural, Pesca y Alimentación y Sociedad Mexicana de Fitogenética, A.C.

Paiaro, V., Oliva, G.E., Cocucci, A.A., \& Sérsic, A.N. (2012). Geographic patterns and environmental drivers of flower and leaf variation in an endemic legume of Southern Patagonia. Plant Ecology \& Diversity, 5(1), 13-25.

Panero, J., \& Villaseñor, J.L. (2008). Asteráceas (Dicotiledóneas). En S. Ocegueda \& J. Llorente-Bousquets (Eds.), Catálogo taxonómico de especies de México. Capital natural de México (Vol. I), Conocimiento actual de la biodiversidad (CD1). Ciudad de México, 
México: Comisión Nacional para el Conocimiento y Uso de la Biodiversidad.

Peterson, A.T., Ball, L.G., \& Cohoon, K.P. (2002). Predicting distributions of Mexican birds using ecological niche modelling methods. International Journal of Avian Science, 144(1), 27-32.

Peterson, A.T. (2003). Projected climate change effects on Rocky Mountain and Great Plains birds: generalities of biodiversity consequences. Global Change Biology, 9(5), 647-655.

Phillips, S.J., Dudík, M., \& Schapire, R.E. (2019). Maxent software for modeling species niches and distributions (Version 3.4.1). Retrieved from http://biodiversityinformatics.amnh.org/open_source/maxent/

Plovanich, A.E., \& Panero, J.L. (2004). A phylogeny of the ITS and ETS for Montanoa (Asteraceae: Heliantheae). Molecular Phylogenetics and Evolution, 31, 815-821.

Robles-Zepeda, R.E., Lozoya-Gloria, E., López, M.G., Villarreal, M.L., Ramírez-Chávez, E., \& MolinaTorres, J. (2006). Montanoa tomentosa glandular trichomes containing kaurenoic acids chemical profile and distribution. Fitoterapia, 80, 12-17.
Soberón, J., \& Peterson, A.T. (2005). Interpretation of Models of Fundamental Ecological Niches and Species' Distributional Areas. Biodiversity Informatics, 2, 1-10.

van Devender, T.R., Sanders, A.C., Wilson, R.K., \& Meyer, S.A. (2000). Vegetation, flora and seasons of the Río Cuchujaqui, a tropical deciduous forest near Alamos, Son. En R.H. Robichaux \& D. Yetman (Eds.), The Tropical Deciduous Forest of Alamos: Biodiversity of a Threatened Ecosystem in Mexico (pp. 37-101). Tucson, Arizona: The University of Arizona Press.

Villa-Ruano, N., Betancourt-Jiménez, M.G., \& LozoyaGloria, E. (2009) Biosynthesis of uterotonic diterpenes from Montanoa tomentosa zoapatle. Journal of Plant Physiology, 166, 1961-1967.

Villa-Ruano, N., \& Lozoya-Gloria, E. (2014). Anti-fertility and other biological activities of zoapatle (Montanoa spp.) with biotechnological application. Boletín Latinoamericano y del Caribe de Plantas Medicinales y Aromáticas, 13, 415-436.

Zhao, K.J., Dong, T.T.X., Tu, P.F., Song, Z.H., Lo, C.K., \& Tsim, K.W.K. (2003). Molecular genetic and chemical assessment of Radix Angelicae (Danggui) in China. Journal of Agricultural and Food Chemistry, $51,2576-2583$.

See Digital Appendix at: / Ver Apéndice digital en: revistas.ucr.ac.cr 